\title{
Hybrid propagation channel modelling for city area land mobile satellite communications
}

\author{
Xiang Li ${ }^{*}$, Rodolphe Vauzelle, Yannis Pousset and Pierre Combeau
}

\begin{abstract}
In this study, a hybrid model is proposed to simulate the realistic channel behavior for urban city land mobile satellite (LMS) communications. It associates the advantages of existing models in that different receiving states are predicted using a deterministic approach, whereas the channel behavior is simulated using adapted statistical laws. Using a geosynchronous satellite transmitter working at $1.5 \mathrm{GHz}$ and a land mobile receiver, the hybrid model is validated for LMS channel. It leads to a high-performance simulation, combining accuracy (global simulation error less than $1 \mathrm{dBW}$ ) and small computation time (gain of about 10,000 times compared to a full deterministic reference model).
\end{abstract}

\section{Introduction}

Satellite communication systems are a valuable alternative to terrestrial systems and supports a wide range of applications, such as mobile radio communications, television transmission, radio localization, satellite Internet, military applications and so on. The land mobile satellite (LMS) channel is an important part of the system and is an ever expanding researching field. Many complex factors may influence this channel. In urban areas, for example, radio wave propagation is highly affected by buildings, terrain shape and other obstacles. Shadowing and multipath effects are present and degrade the LMS channel performance.

In order to predict channel behaviors in different propagation conditions, channel models are among the most useful solutions. They are developed to accompany or even to replace measuring campaigns which turn out to be time- and cost-consuming. These models rely on the understanding of channel characteristics and on simplifications, privileging either computation time or accuracy.

Three main families exist in LMS channel modelling: deterministic, statistical and hybrid models. The most used deterministic models are those based on geometric optics $[1,2]$. They simulate detailed channel properties and can be directly applied to any 3D maps. However,

\footnotetext{
* Correspondence: xiangli496@foxmail.com Laboratory XLIM-SIC, UMR CNRS 6172, University of Poitiers, SP2MI-T'el'eport 2-Boulevard Marie et Pierre Curie-BP 30179, 86962 Futuroscope Chasseneuil Cedex, France
}

their main drawback is the computation time due to high complexity in ray tracing algorithms.

Statistical models, on the other hand, are based on measured or simulated data and associated channel behavior analysis. Statistical parameters can be deduced from these data and fed into random generators to simulate the channel. The main advantage of statistical models is the low computation time. Typical LMS channel models can be found in the literature [3-5]. In general, statistical models are often validated for specific area types in a global manner, e.g., a single parameter setup is used in a city area. This may limit the accuracy and the generalization of these models.

Being able to combine the advantages of the two families above, hybrid models have attracted the attention of many researchers. In [6], the channel modelling was based on a conditional Rice distribution. Using a ray tracing technique (deterministic), Rician parameters (statistical) were proved to be related to building height, street width and other terrain parameters. In [7-9], virtual propagation environments can be generated according to physical-statistical environment classification. This study, as well as [10], shows that different receiving states related to Line-Of-Sight (LOS) and Non-Line-OfSight (NLOS) may exist and can be modelled by a Markov chain simulator, whereas the global signal variations within each state was predicted by the Loo distribution [3]. The notion of receiving states was also reported in [11]. \\ 基 Springer}


Our work belongs to the hybrid family. It is partially inspired by several existing works cited above and makes new contributions. For example, instead of generating virtual environments, we use some of the environment characteristics in $[6,12]$ in order to classify our deterministic city environments. Different receiving states, as proposed in $[7,10,11]$ are also observed in our simulated deterministic signals. Here, the novelty of our study lies in the deterministic state-locating method. Moreover, the NLOS severe state ("blocked"), which was not considered in specific applications like $[7,11]$, is taken into account and thus makes the model general. Statistical parameters are estimated and validated according to different environment classes and satellite positions. The proposed model gives both computation efficiency and satisfying accuracy.

The remainder of this article is organized as follows: Section 2 describes the basis of the proposed model. The design of the hybrid model is presented in Section 3. In Sections 4 and 5, the model is implemented and its performance is evaluated with respect to reference data in terms of accuracy and computation time. Finally, we draw conclusion in Section 6.

\section{Basis of the proposed model}

In this section, the LMS channel behavior is analyzed. Based on the multipath nature, a deterministic statelocating method is proposed, followed by a state-specific statistical modelling. In this study, we use the Ergospace software to simulate LMS communications in different configurations. This commercial software is based on ray tracing and is purely deterministic. 3D maps, satellite transmitter, antenna type and receiver course are among its input data. It has to be noted that we only use Ergospace as a tool but the concept is entirely independent of it and can be implemented using any ray tracing software.

\subsection{LMS channel analysis}

The simulations in this study are configured with a geosynchronous satellite working at $1.5 \mathrm{GHz}$ ( $L$-band) at different longitudes and a land mobile receiver travelling through city environments, e.g., dense urban, urban and suburban areas of a city. Figure 1a shows a typical simulated deterministic signal in terms of received power (in $\mathrm{dBW}$ ) along a receiver course of about $600 \mathrm{~m}$ through the district called "Carmes", a dense urban area of Toulouse (France). The Effective Isotropic Radiated Power (EIRP) is $14.77 \mathrm{dBW}(30 \mathrm{~W})$. Figure $1 \mathrm{~b}$ shows the associated cumulative density function (CDF).

Figure $1 \mathrm{a}$ and $1 \mathrm{~b}$ exhibit three receiving states. The LOS state can be found around $-168 \mathrm{dBW}$, with nearly stable signal power. The NLOS weak state lies between -187 and $-178 \mathrm{dBW}$ with more signal variations. The NLOS severe state is largely distributed from -240 to 187 $\mathrm{dBW}$, indicating severe fading and rapid signal fluctuations. These observations are conform to previous studies like $[7,11]$ and lead to the studies of the next section.

\subsection{Deterministic state-locating}

In this article, we take advantage of ray tracing-based software to propose a deterministic state-locating technique.

We first assume that the occurrence of these different states is related to the multipath nature. It is known that the origin of the multipath effect is interaction between wave and obstacle. For a given path, radio wave may undergo $p$ reflections (R) and/or $q$ diffractions (D) before arriving at the receiver. These numbers are

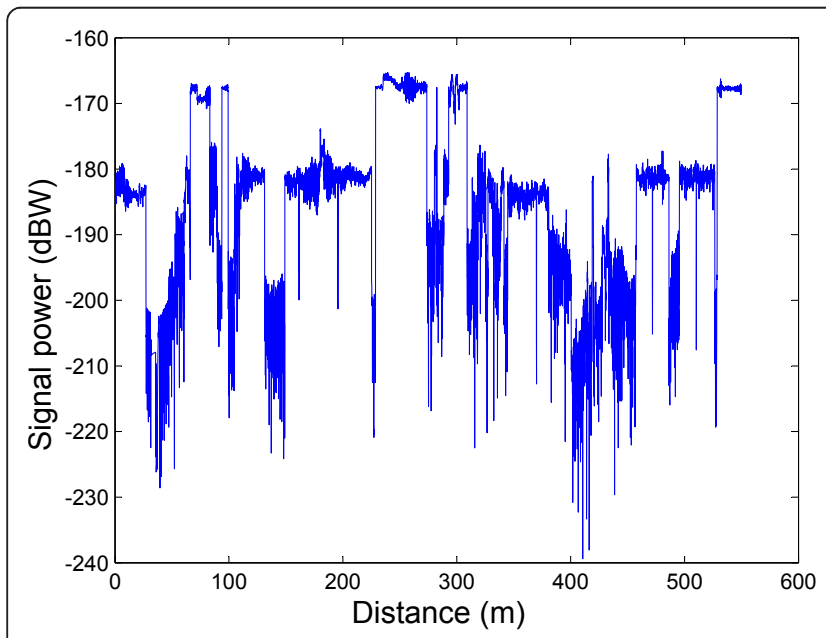

(a)

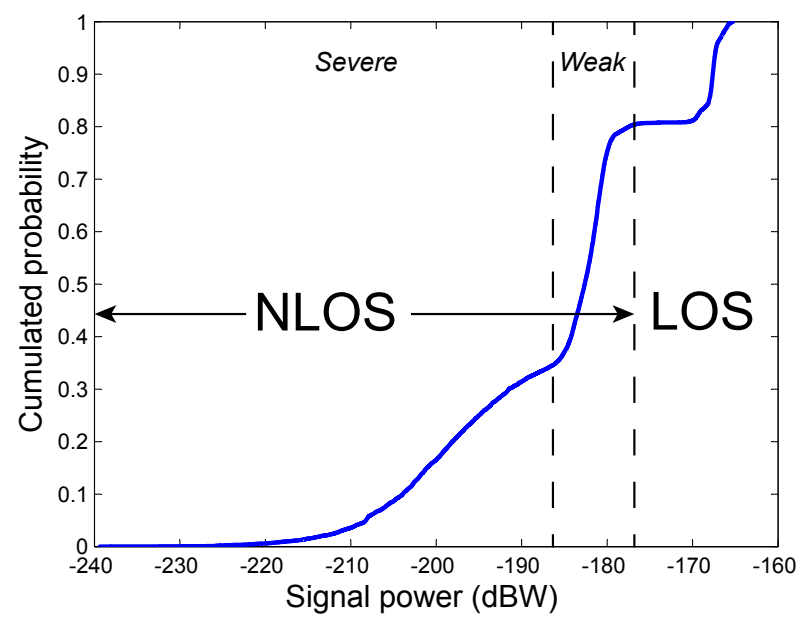

(b)

Figure 1 Example of a simulated signal in a dense urban environment of Toulouse: (a) signal envelop; (b) CDF of the signal 
recorded by ray tracing software as simulation output. If we consider the combination of interactions (CI), this path can be tagged as $p \mathrm{R} q \mathrm{D}$. Also, it has to be noted that in satellite communications, the maximum number of interactions to be considered is two for reflection and one for diffraction. In fact, the signal will be too attenuated over this limit and the corresponding path is not calculated in the simulation. This limit is validated by Ergospace in collaboration with CNES (the French Space Agency) based on measured data and used in existent works like [11]. Hence, six types of $\mathrm{CI}$ are valid: OROD (direct path), 1R0D (single reflected path), 2ROD, 0R1D, 1R1D and 2R1D.

Next, for a sample point, we can note a CI group by considering the CI types of the arriving path. For example, a sample point is noted "0R0D + 1R0D + 2R1D" if all received paths are of these three CI types. Note that since the method is based on multipath nature, the number of paths of the same CI is not considered. Now if each CI has a numeric weight, a CI group becomes a sum noted as $\Sigma_{C I}$. It reflects the multipath nature on this sample point.

The choice of numeric weights is based on two criteria. Firstly, the CI associated with less attenuation will be assigned with a greater value. The order of magnitude for CIs can be easily determined: paths with more interactions will lose more power, so OROD is greater than 1R0D; diffractions result in greater attenuation than reflections [13], so 1R0D is greater than 0R1D. Secondly, we have chosen a series of binary weights in order to make unique correspondence between $\Sigma_{\mathrm{CI}}$ values and CI groups. Table 1 shows the chosen weights.

For example, a CI group of "0R0D + 1R0D + 2R1D" corresponds to $\Sigma_{\mathrm{CI}}=32+16+1=49$ without ambiguity, according to this table. All possible values of $\Sigma_{\mathrm{CI}}$ range from 1 to 63 .

In this manner, each sample point is associated with a $\Sigma_{\mathrm{CI}}$ value, giving birth to the $\Sigma_{\mathrm{CI}}$ evolution along the received signal. The $\Sigma_{C I}$ evolution can be used to segment and locate different receiving states: we sort the $\Sigma_{\mathrm{CI}}$ evolution from 1 to 63 (Figure $2 \mathrm{a}$ ) and reorganize the signal sample points accordingly. As a result, sample points with similar multipath nature are now next to each other. Figure $2 b$ shows the reorganized signal samples according to Figure 2a.

In this figure, each point at which $\Sigma_{C I}$ changes value can be considered as a divide of two different multipath natures. However, it may not be necessary to segment the signal in such detail, as we are mainly interested in

Table 1 Types of $\mathrm{Cl}$ and associated weights

\begin{tabular}{lllllll}
\hline Cl & OROD & 1R0D & 2R0D & OR1D & 1R1D & 2R1D \\
\hline Weight & 32 & 16 & 8 & 4 & 2 & 1 \\
\hline
\end{tabular}

locating different receiving states (LOS, NLOS weak and NLOS severe). Indeed, the LOS and NLOS states can be separated by $\Sigma_{\mathrm{CI}}=32$ (the blue line in Figure 2) because it indicates the presence of a direct path. In other words, all sample points with $\Sigma_{C I} \geq 32$ are in LOS state.

As for NLOS severe and weak areas, the segmentation can be done for $\Sigma_{C I}=16$. This is justified because the value 16 corresponds to $1 \mathrm{R} 0 \mathrm{D}$, in other words, paths reflected only once and not diffracted. This is the predominant component in the absence of the direct path, as suggested in the studies $[11,13]$. As a result, all sample points with $\Sigma_{\mathrm{CI}}<16$ are in NLOS severe areas, while others $\left(16 \leq \Sigma_{\mathrm{CI}}<32\right)$ are in NLOS weak areas. Hence, using the proposed $\Sigma_{\mathrm{CI}}$ method, the signal can be segmented in three states in a deterministic manner.

Another conclusion can be drawn from the above analysis: in order to locate these states, we can simply rely on the presence of two CIs: 1ROD and 0R0D. This can drastically reduce the ray tracing complexity. Unlike the purely deterministic approach which allows up to two reflections and one diffraction, the state-locating only need to allow one reflection. The gain in computation time will be given in Section 5.2.

\subsection{State-specific statistical modelling \\ 2.3.1 Principles}

It can be observed in Figure 2 that the received signal exhibits different degrees of large and small-scale fading variation according to the receiving state. Thus, these different degrees of variation by receiving state induce different degrees of stationarity. So, for each state, we aim at finding a suitable statistical law to model the signal behavior. In other words, we think that the pertinence of the chosen statistical distribution depends on the degree of stationarity of the signal. In order to quantify the stationarity, we propose to study three aspects of the received signal by state:

- the mean signal level (first order statistic);

- the standard deviation (second order statistic);

- the covariance statistic within a state.

For the first two quantities, we use the well known sliding-window technique with a window size of 125 samples (about $48 \lambda$ according to [14]) to calculate the local mean and standard deviation in each window. So, more stationary the signal is, more constant these two quantities will be.

Concerning the covariance, we define it by the following expression:

$$
R_{X X_{\tau}}(\tau)=\operatorname{Cov}\left(X, X_{\tau}\right)=\frac{\sum_{i=1}^{N} x_{i} x_{i+\tau}-\sum_{i=1}^{N} x_{i} \sum_{i=1}^{N} x_{i+\tau}}{N}
$$


(a)

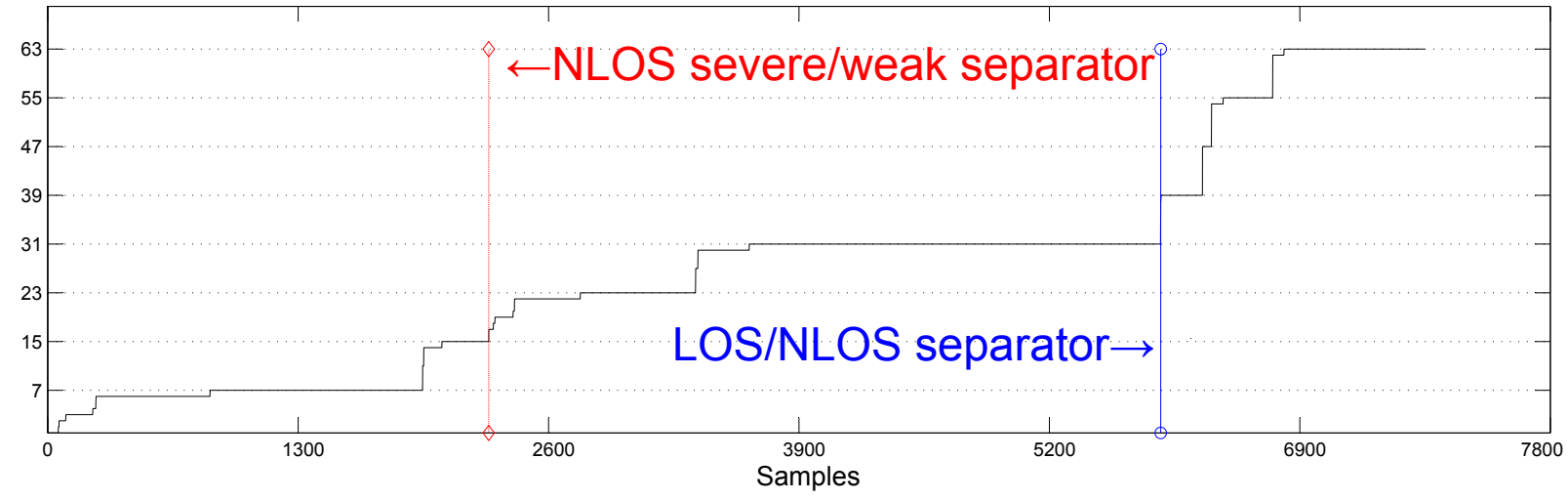

(b)

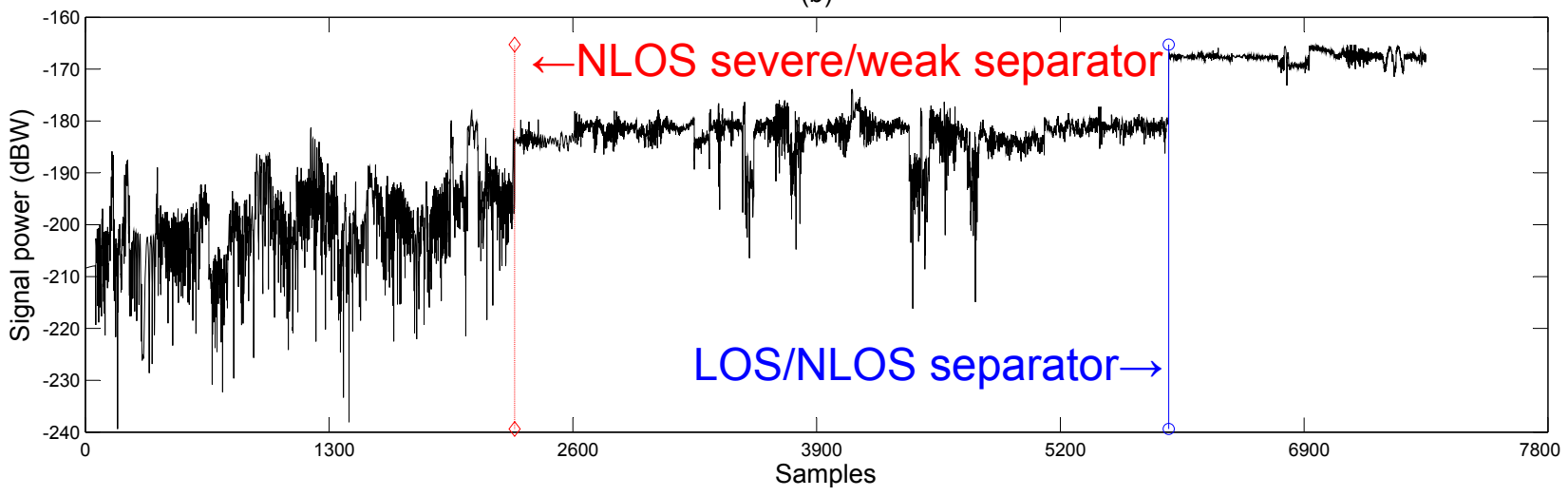

Figure 2 Application of the $\Sigma_{\mathrm{Cl}}$ segmentation method: (a) sorted $\Sigma_{\mathrm{Cl}}$ evolution; (b) associated signal.

where $X$ and $X_{\tau}$ are two windows of the received signal, each containing $N$ samples $x_{i}$ and $x_{i+\tau}$ such that $i=1,2, \ldots$, $N$. These two windows are shifted with an offset noted $\tau$. Moreover, in our study context, we again consider a window size of $48 \lambda$ (corresponding to about 125 samples).

Thus, more stationary the signal is by state, more stable the covariance will be for a given offset $\tau$ in different window pairs $\left(X, X_{\tau}\right)$. We conclude on the quasi stationarity of the signal if these covariances are appreciably equal.

\subsubsection{Signal stationarity in different states}

Figure $3 \mathrm{a}$ and $3 \mathrm{~b}$ indicate the local mean and standard deviation of the sorted signal in Figure $2 b$, respectively. Compared to the NLOS severe and weak states, the LOS state shows greater mean values with very small dynamic range. If we calculate the standard deviation of these local mean values within a state, we find $0.61,1.92$ and 4.98 for LOS, NLOS weak and NLOS severe, respectively. For local standard deviation values, the dynamic ranges are found to be $0.36,1.73$ and 1.19 , respectively.

Figure 4 indicates the covariance analysis with two offsets: 62 samples $(\approx 24 \lambda)$ and 94 samples $(\approx 36 \lambda)$ applied to the same signal. For example, Figure $4 \mathrm{a}$ means that the shifted window $X_{\tau}$ is defined with an offset $\tau \approx 24 \lambda$ with respect to the fixed window $X$.

It can be observed that, compared with the NLOS states, the LOS state still has very small covariance values (generally close to 0 ) with very small variation. Globally, we find an averaged standard deviation value (calculated from all the 125 different offsets) of 0.27 for LOS, compared to 3.04 for NLOS weak and 9.02 for NLOS severe.

\subsubsection{Statistical model for the LOS state}

From the above study, we can conclude that the signal is quasi stationary in the LOS state. We hence propose to consider a constant global mean $\bar{A}$ received power in LOS areas as the large-scale parameter. Then, we need to find a statistical model for the small-scale fading (which will be added to $\bar{A}$ ). This refers to a procedure called law recognition detailed in [15]. In the context of satellite communications, the Nakagami-m model [16] turns out to be the most adequate and robust. Equation (2) indicates its probability density function:

$$
p(r)=\frac{2 m^{m} r^{2 m-1}}{\Gamma(m) \Omega^{m}} e^{-(m / \Omega) r^{2}}, \quad m \geq \frac{1}{2}, \quad r \geq 0
$$




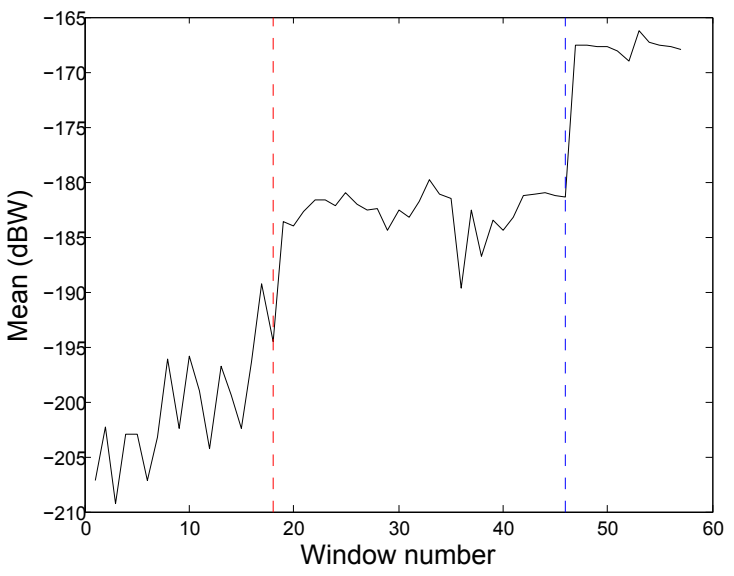

(a)

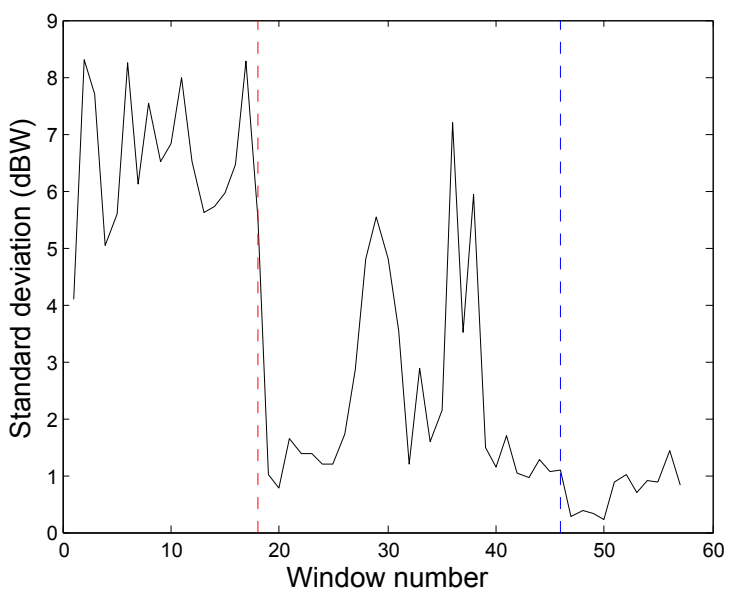

(b)

Figure 3 Statistical analysis of the simulated signal in Figure 2b: (a) First order; (b) second order. Red line: NLOS severe/weak separator; Blue line: NLOS/LOS separator.

where $\Omega=\mathrm{E}\left\{r^{2}\right\}=\overline{r^{2}}$ is the signal variance and $m$ the fading degree parameter. Therefore, three statistical parameters are necessary for LOS areas: constant global mean $\bar{A}, m$ and $\Omega$. For the LOS segment in Figure 2, the signal modelled by Nakagami- $m$ setup results in a RMSE of $0.48 \mathrm{dBW}$ (using CDF comparison) with respect to the original signal.

\subsubsection{Statistical model for the NLOS states}

The signal behavior for NLOS states is quite different from LOS state: it has low mean power and the local mean varies quickly. As a result, it can be intricate to separate the large-scale fading within an acceptable confidence interval. According to maximum likelihood estimation, the lognormal model (also used in [7]) seems to give the best fit to describe the large- and small-scale fading together. Accordingly, two pairs of parameters should be used, one for NLOS severe $\left(\mu_{s}\right.$ and $\left.\sigma_{s}\right)$ and the other for NLOS weak $\left(\mu_{w}\right.$ and $\sigma_{w)}$. For the NLOS severe and NLOS weak segments in Figure 2, the lognormal setup results in RMSEs of 0.84 and 1.48 $\mathrm{dBW}$, respectively. This is much better than the Nakagami- $m$ setup with RMSEs of 1.95 and $2.70 \mathrm{dBW}$.

\section{Model design}

Based on the principles discussed in the previous section, we present the model's workflow in this section. It works in two phases: the learning phase for parameter estimation and the application phase to actually simulate the "hybrid" signal.

\subsection{Learning phase: parameter estimation}

The learning phase begins by determining adequate statistical parameters of the previous laws (Nakagami and lognormal) in different receiving states. The basis of this step has been covered in Sections 2.2 and 2.3. In practice, we first choose a representative environment as reference scene and launch a purely deterministic simulation with Ergospace allowing a maximum of two (a) Offset $=24 \lambda$

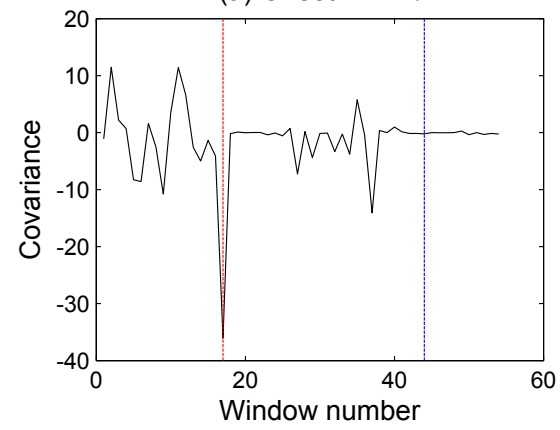

(b) Offset $=36 \lambda$

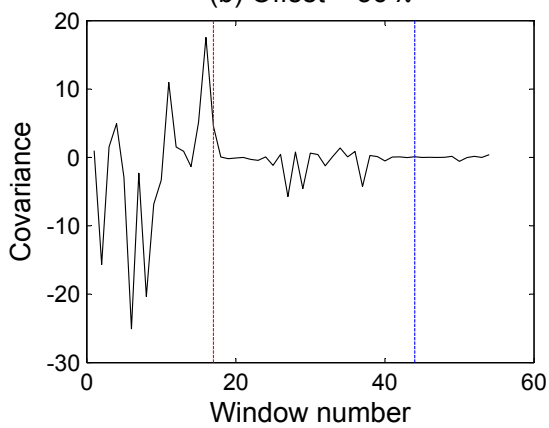

Figure 4 Covariance statistics using different offsets. 
reflections and one diffraction (the "classical" configuration). Secondly, the $\Sigma_{C I}$ method is applied to the simulated deterministic signal in order to find different states. Within each state, statistical parameters are estimated using maximum likelihood estimation for the corresponding law (Nakagami- $m$ or lognormal). The parameter estimation is done only once per environment type.

It is also important to note that these parameters are valid for a given configuration. According to [7], the main factors of a configuration are the transmitter position and the environment type. Concerning the former, different geosynchronous satellite positions are taken into account in this study. As for the environment type, a classification approach is necessary to regroup different environments according to their similarity. This is done by finding terrain parameters having a strong impact on radio wave communications. Different classifications can be found in the literature. In our study, we have considered the following factors:

- Building density $(S)$ : percentage of area covered by buildings

- Meal building height $(\bar{h})$

- Standard deviation of building height $\left(\sigma_{h}\right)$

In fact, these are the three most important factors in city area communications $[17,18]$ and are also used in generating virtual environments in simulations [12]. We have studied a total of six districts of Toulouse (Figure 5 ) and the classification result is given in Table 2. From this table, it can be observed that the building density $S$ (\%) decreases from dense urban to suburban. The mean building height $\bar{h}$ in dense urban is about $20 \mathrm{~m}$. Urban and suburban areas share a smaller but similar mean height of about $16 \mathrm{~m}$. This means the receiving areas become more open from dense urban to suburban areas.

On the other hand, the standard deviation $\sigma_{h}$ is low in dense urban areas, meaning that the buildings are of similar heights. However, from urban to suburban, the building height become more and more variable, indicating a great diversity of building types. $\sigma_{h}$ is also the main difference between urban and suburban areas.

\subsection{Application phase: hybrid simulation}

The hybrid model now proceeds to its application phase which actually simulates the signal, as indicated in Figure 6.

In this step, we choose an application scene in the same environment class as the reference scene. As suggested in Section 2.2, the deterministic module now works in an optimized configuration with a maximum of one reflection. It thus locates only the positions of the three states along a given receiver course. The signal samples are simulated through random generators configured according to statistical parameters estimated in

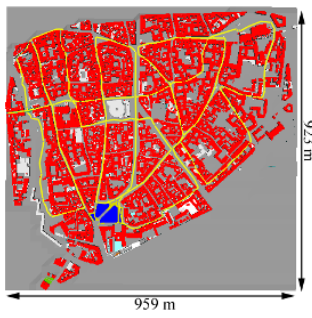

(a) Dense urban (reference)

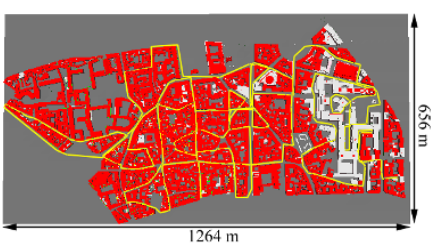

(b) Dense urban (application)

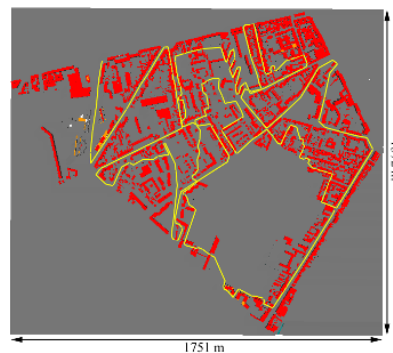

(e) Suburban (reference)

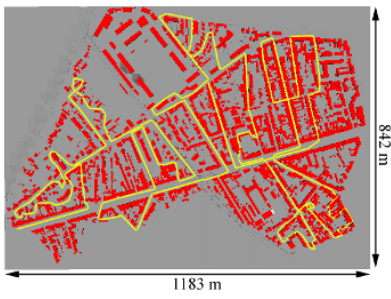

(c) Urban (reference)

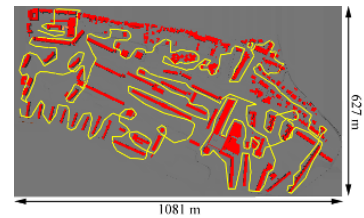

(f) Suburban (application)

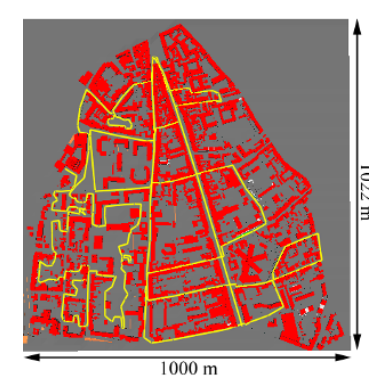

(d) Urban (application)

Figure 5 City environments of Toulouse: (a) Carmes; (b) Capitole; (c) St-Agne; (d) St-Michel; (e) Arènes; (f) Empalot. Receiver courses are marked in yellow. 
Table 2 Environnement classification of six city areas

\begin{tabular}{llll}
\hline Environnement & $\boldsymbol{S}(\%)$ & $\bar{h}(m)$ & $\boldsymbol{\sigma}_{\boldsymbol{h}}(\mathrm{m})$ \\
\hline Dense urban & & & \\
$\quad$ Carmes & 50 & 19 & 9 \\
$\quad$ Capitole & 55 & 20 & 10 \\
Urban & & & \\
$\quad$ St-Agne & 27 & 14 & 13 \\
$\quad$ St-Michel & 35 & 18 & 16 \\
Suburban & & & \\
Arènes & 22 & 14 & 26 \\
Empalot & 17 & 16 & 24 \\
\hline
\end{tabular}

the learning phase for a given environment class and satellite position. For example, the samples of the LOS state will be generated using the Nakagami- $m$ random generator, overlapped onto a constant global mean. The NLOS states are generated agreeably to two different lognormal distributions (NLOS weak and NLOS severe). Finally, all generated samples are associated with sample locations indicated by the deterministic module.

\section{Learning phase implementation}

In order to apply the hybrid model, we choose two representative environments of each class in Figure 5, one serves as reference scene and the other as application scene. The model's learning phase is presented in the section.

\subsection{Global state occurrence}

Figure 7 shows the state occurrence rate for 13 different satellite positions in each of the three environment types of Figure 5a, c and 5e. The position of a geosynchronous satellite is indicated by its longitude. Note that we use the notion of longitude in our study because it is a directly configurable satellite-related parameter. Seen from Toulouse (latitude: $43^{\circ} 37^{\prime} \mathrm{N}$, longitude: $1^{\circ} 26^{\prime} \mathrm{E}$ ), from $300^{\circ}$ to $360^{\circ}$, it is like that the satellite emerges and "goes up" until the highest position. Then, from $0^{\circ}$ (or $360^{\circ}$ ) to $60^{\circ}$, it gradually "goes down" and disappears.

\subsubsection{Dense urban areas}

We observe that in dense urban areas with a low angle, e.g., $300^{\circ}$, a large part $(79.6 \%)$ of the received signal is

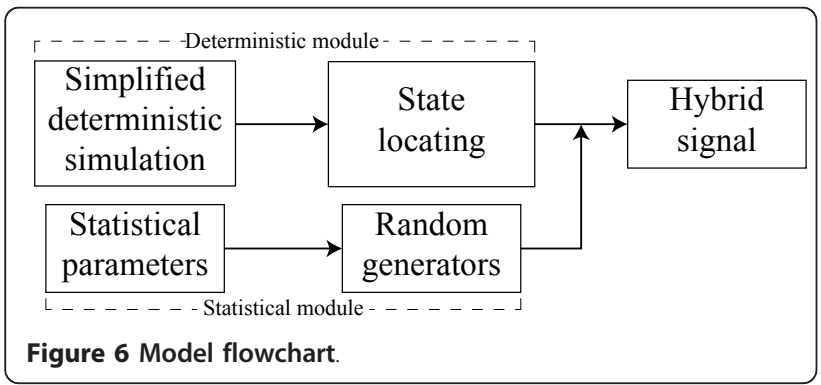

found in NLOS severe state, whereas LOS and NLOS weak states are less often present. These two states gradually increase with the longitude. At a high angle like $360^{\circ}$, the occurrence of the three states seems to be well balanced. Also note the increasing trend of NLOS weak state for dense urban areas. In this case, radio waves have more chance to arrive (through simple reflection) at the receiver when the satellite is high. Otherwise, multiple reflection is needed and the sample point is in NLOS severe state.

\subsubsection{Urban and suburban areas}

The state occurrence rates of these two areas are in general very different from dense urban areas. Even in the worst case $\left(300^{\circ}\right)$, we find a minimum of $42.2 \%$ of LOS state. This is mainly due to the difference in building density $(S)$ and mean building height $(\bar{h})$.

These two areas share similar state occurrence statistics. This can be explained in referring to Table 2: urban and suburban areas are globally similar in terms of $S$ and $\bar{h}$ while the factor $\sigma_{h}$ makes them different. But as satellite transmitters are very far from the Earth, the building height variation becomes a minor factor on the state occurrence.

\subsection{Statistical behavior in each state \\ 4.2.1 LOS state}

The Figure 8 shows that the global mean power $\bar{A}$ increases with the longitude and seems to be seldom affected by the environment class.

It has been pointed out in Section 2.3 that the signal mean power is considered to be constant due to the quasi-invariant transmitter-receiver separation. This is only true for a given satellite position. In fact, a change in transmitter position also modifies the angle of departure (off-nadir angle) and the angle of arrival (site angle or elevation) and thus results in different antenna gains. It is therefore necessary to study how the off-nadir angle and the elevation angle change with longitude. We find that due to the great distance of a geosynchronous satellite, the off-nadir angle does not change significantly. The transmitter gain is $12.9 \mathrm{~dB}$ at $300^{\circ}$ and 12.4 $\mathrm{dB}$ at $360^{\circ}$. The receiver gain, on the other hand, can be determined using the antenna radiation pattern and the elevation angle. Figure 9 shows the radiation pattern of the studied antenna, with the elevation range (between $11.7^{\circ}$ and $39.7^{\circ}$ as calculated according to the longitude range) marked in gray. Generally speaking, higher satellite elevations (closer to zenith) result in higher receiver antenna gains. If we calculate and remove the influence of antenna gains (both transmitter and receiver), the curves in Figure 8 become flat with the signal power around $-176 \mathrm{dBW}$, corresponding to the signal power received through freespace. 


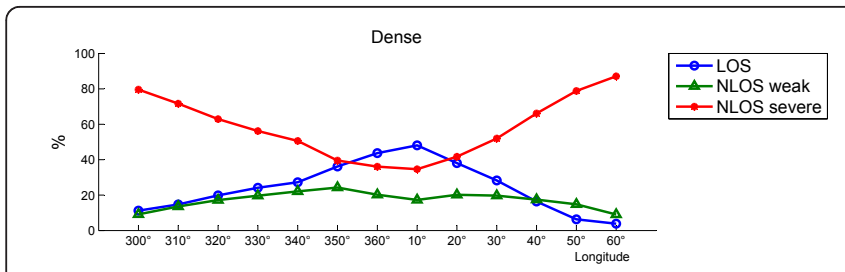

(a)

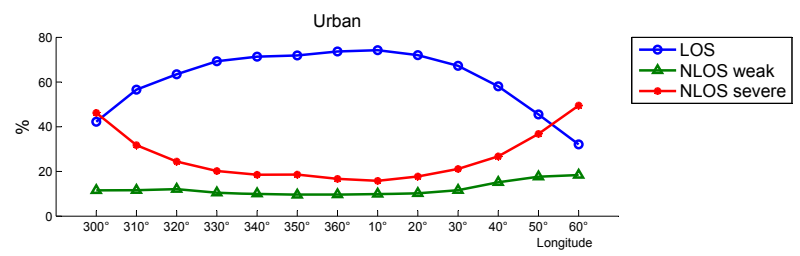

(b)

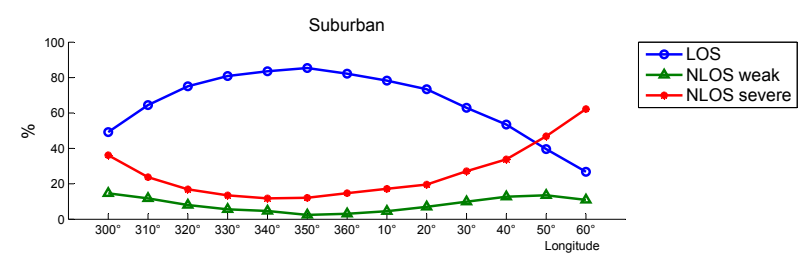

(c)

Figure 7 State occurrence rate: (a) dense urban; (b) urban; (c) suburban.

Concerning the small-scale fading, Figure 10 indicates the evolutions of Nakagami- $m$ parameters. When the satellite goes higher, we find $m$ increase (less severe fading) while $\Omega$ decreases (less signal variations). Both observations confirm that when the transmitter is high, the influence of multipath decreases.

The influence of environment class is mostly reflected by signal variance, where dense urban areas have in general greater $\Omega$ values. In fact, more paths are created in dense urban areas due to tightly surrounding buildings.

\subsubsection{NLOS severe state}

The parameter evolutions for NLOS severe state are indicated in Figure 11. They seem to be similar in urban and suburban areas, but different in dense urban areas.

This difference is explained by the Figure 7. It shows that the state occurrence rates in dense urban environment is clearly different from those in urban and suburban ones. In particular, the occurrence of the NLOS severe state is significantly more important. This induce different dominant propagation phenomena: in the

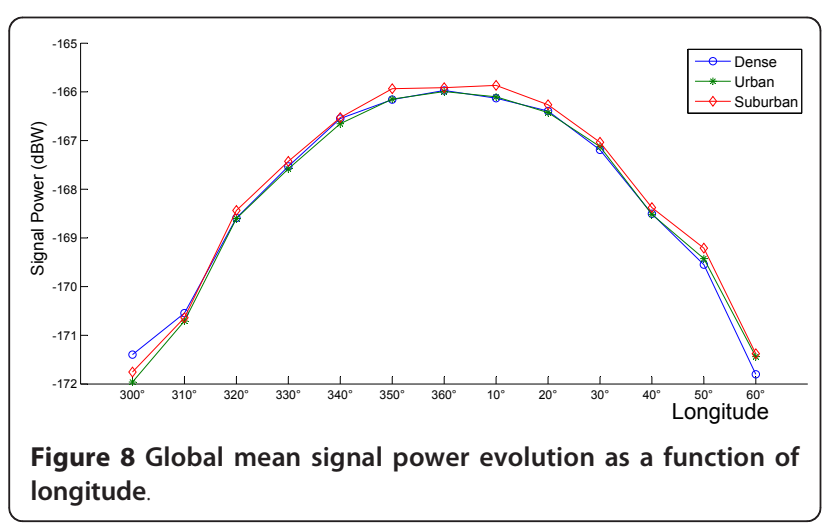

dense urban case, the probability of building blockage is very important as the environment is highly cluttered. Note that the occurrence of the NLOS severe state decreases for high satellite longitudes.

Similar signal behaviors were observed in studies like [19]. In this article, the authors considered the Loo distribution to model the experimental data and defined three states named "LOS", "heavy shadow" and "light shadow" in different environments. They showed that the occurrence of the heavy shadow (corresponding to NLOS severe in our work) state and the channel model parameters are very different in dense urban areas.

\subsubsection{NLOS weak state}

We find greater values for both $\mu_{w}$ and $\sigma_{w}$ in dense urban areas (with respect to urban and suburban). This again can be explained by the abundance of multipath. More received paths contribute to higher signal power but also more rapid fluctuations.

However, for a certain environment class, the evolution of $\mu_{w}$ and $\sigma_{w}$ with respect to the longitude does not seem to be consistent, as shown in Figure 12. As no clear conclusions may be drawn from the data, detailed study is needed in the future to understand the statistical behavior of this transitional state.

Remark. From Figures 8, 10, 11 and 12, we see that the statistical parameters globally evolve in a parabolic manner. One possibility to process data is to apply polynomial fitting to these curves in a least-square sense. As a result, statistical parameters are modelled by polynomial laws and can be calculated for any longitude. In the upcoming application phase, however, as the state occurrence rate and the statistical parameter evolutions all have a certain symmetric behavior, we propose to consider only three representative satellite positions: $300^{\circ}$ (low angle), $330^{\circ}$ (mid angle) and $360^{\circ}$ (high angle). 


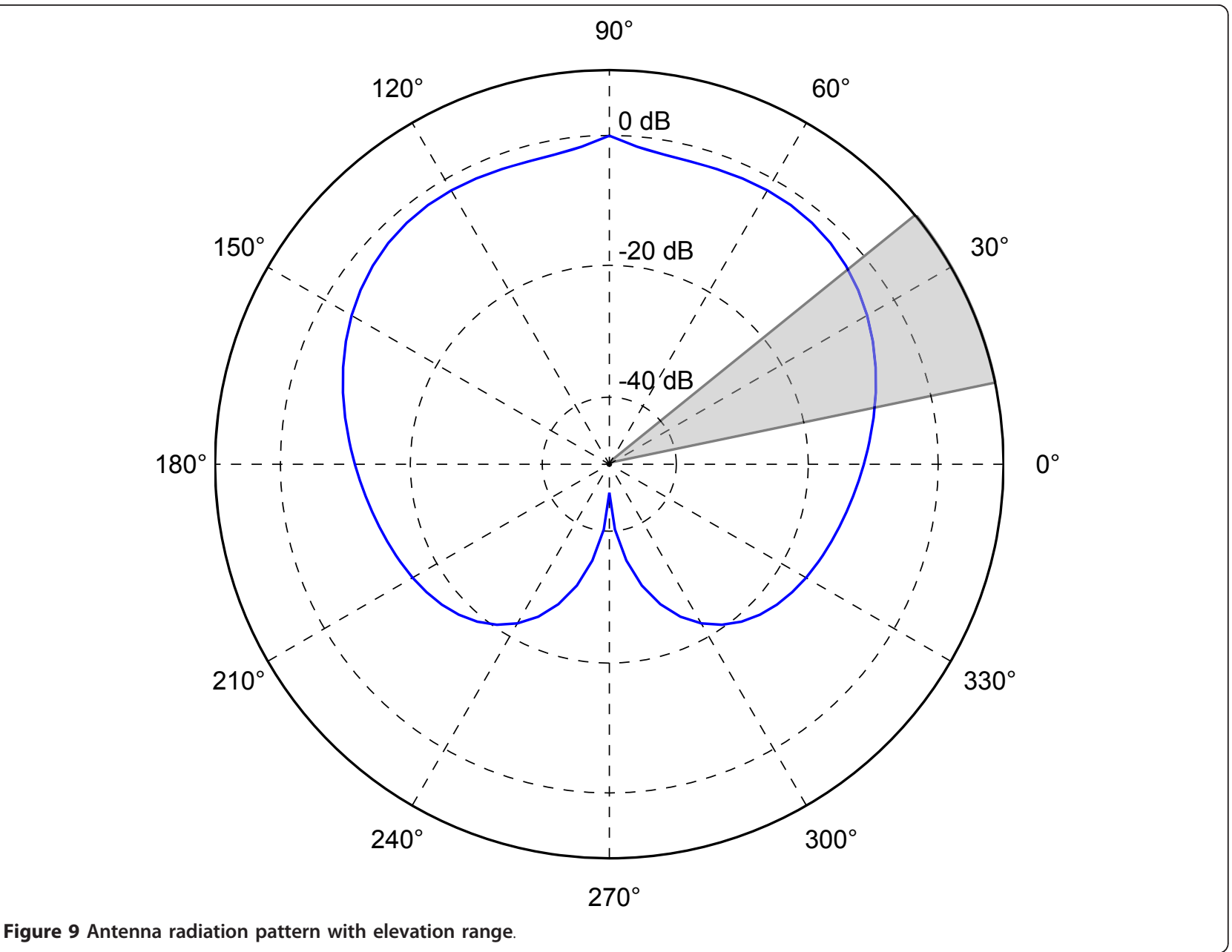

Statistical parameters are calculated using the fitted coefficients and listed in Table 3.

As Table 3 indicates, urban and suburban areas share similar statistical parameters. This follows well the discussions based on Figures 8, 10, 12 and 11 or Table 2. It is possible to simplify further the hybrid model if these two environments are regrouped by taking the mean value of related parameters in Table 3, provided that the accuracy is not greatly reduced. Further study is made in Section 5.1.

\section{Application phase and performance evaluation}

In the application phase, the hybrid model actually simulates the received signal, as described in Section 3.2. The application scenes of Figure $5 b, d$ and $5 f$ are used. This section presents the accuracy and computation time evaluations for the model.

\subsection{Accuracy evaluation}

In order to estimate the simulation accuracy, we plot the CDF of the simulated hybrid signal. The reference is the
CDF of the purely deterministic signal simulated by Ergospace with classical 2R1D configuration along the same receiver course in the application scenes. Moreover, CDFs of the regrouped hybrid signals are plotted for urban and suburban environments using averaged statistical parameters of there two environment types in Table 3 . All the simulations results are indicated in Figures 13, 14 and 15.

In order to quantify the simulation quality, we calculate the root mean square error (RMSE) in terms of $\mathrm{dBW}$ with respect to the deterministic CDF. The results are listed in Table 4.

For dense urban areas, it can be observed that the hybrid signal closely follows the deterministic signal. The RMSEs for $330^{\circ}$ and $360^{\circ}$ are 1.87 and $1.58 \mathrm{dBW}$, respectively. As for $300^{\circ}$, a RMSE of $3.55 \mathrm{dBW}$ is relatively high, it is mainly due to the difference of the NLOS tail in Figure 13a.

The hybrid simulations are highly accurate as well in urban areas. The RMSEs for modelled hybrid signals are found to be $1.83,0.92$ and 0.90 for $300^{\circ}, 330^{\circ}$ and $360^{\circ}$, respectively. Regrouped hybrid signals, on the other 


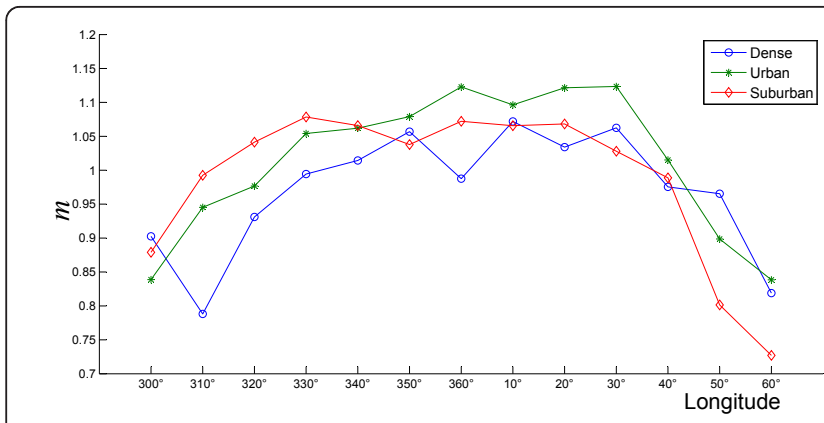

(a)

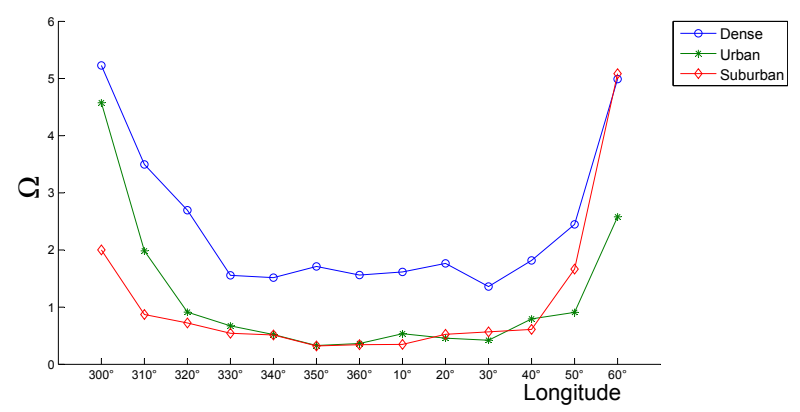

(b)

Figure 10 Nakagami- $m$ parameter evolutions: (a) $m$; (b) $\Omega$

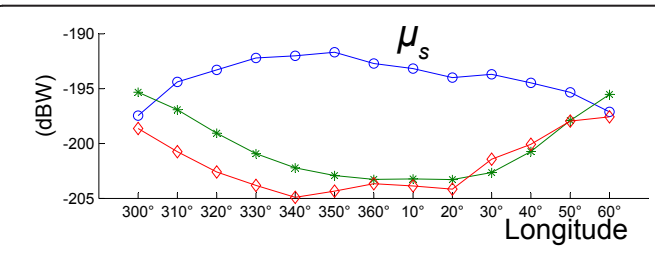

(a)
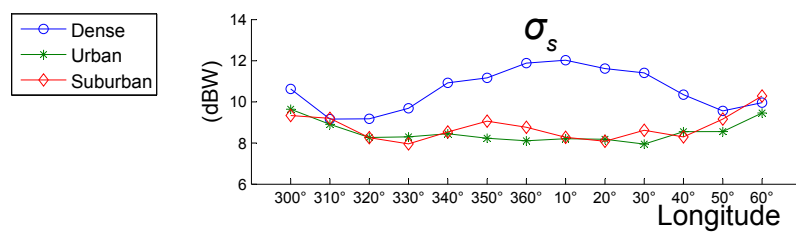

(b)

Figure 11 Lognormal parameter evolutions in NLOS severe state: (a) $\mu_{s}$ (b) $\sigma_{s}$

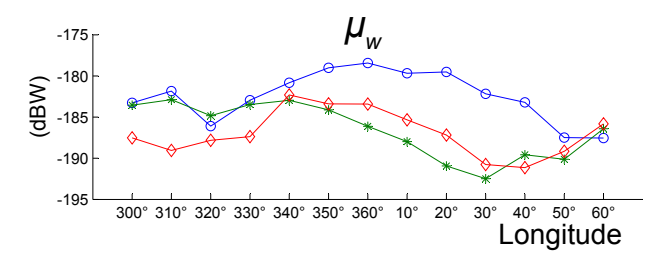

(a)

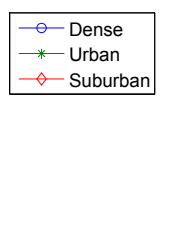

$\sum_{2}^{8}{ }_{4}^{6}$

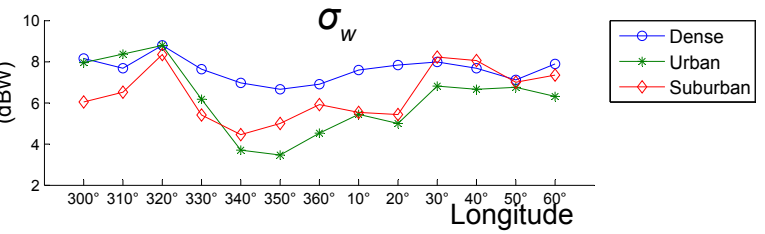

(b)

Figure 12 Lognormal parameter evolutions in NLOS weak state: (a) $\mu_{w i}$ (b) $\sigma_{w}$.

Table 3 Statistical parameters

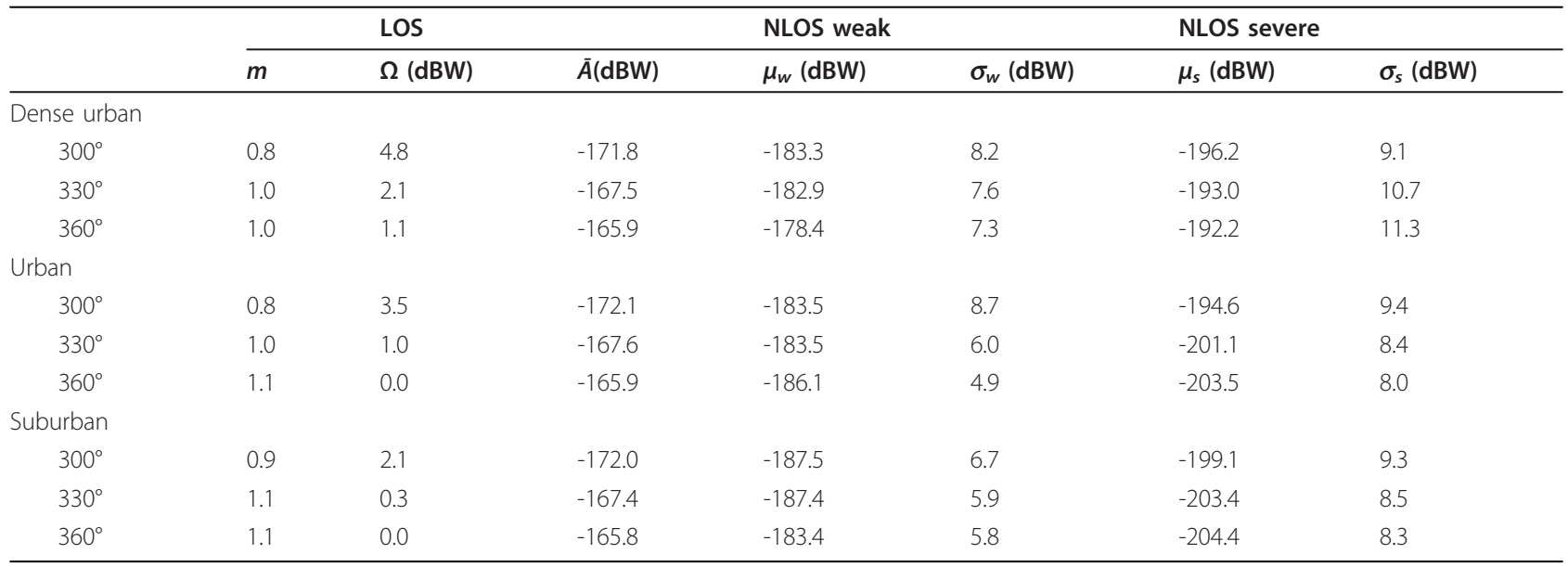




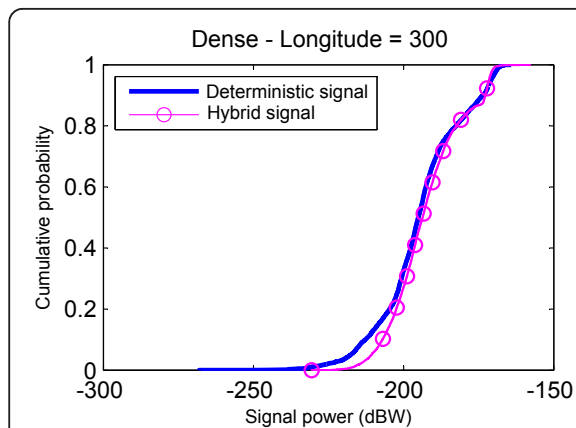

(a)

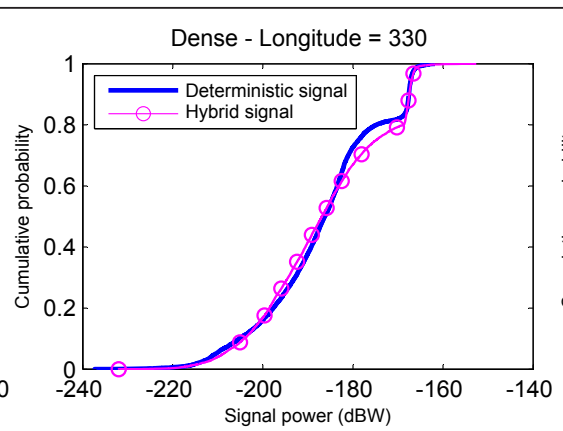

(b)

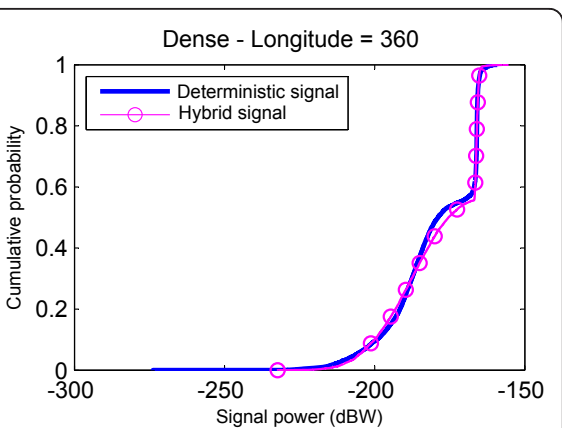

(c)

Figure 13 CDF comparison of dense urban ("Capitole") simulation results: (a) $300^{\circ}$; (b) $330^{\circ}$; (c) $360^{\circ}$.

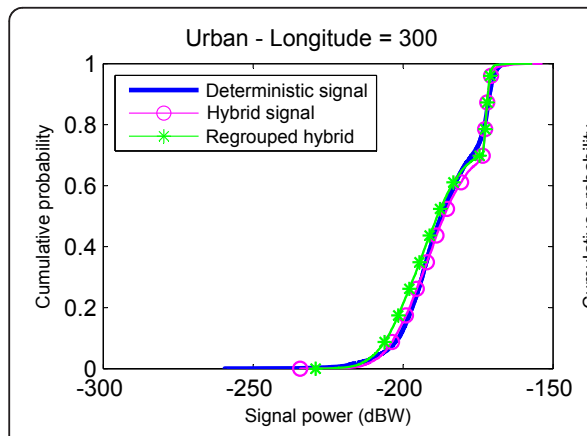

(a)

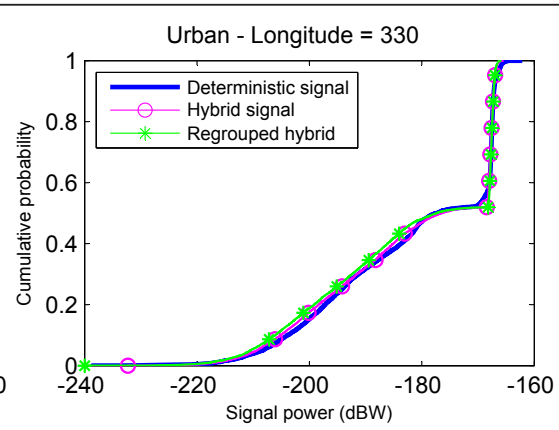

(b)

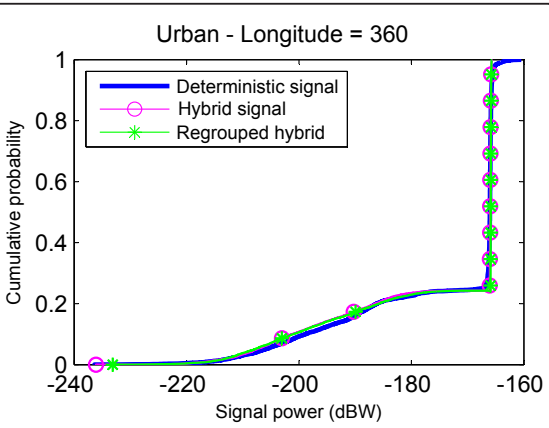

(c)

Figure $14 \mathrm{CDF}$ comparison of urban ("St-Michel") simulation results: (a) $300^{\circ}$; (b) $330^{\circ}$; (c) $360^{\circ}$.

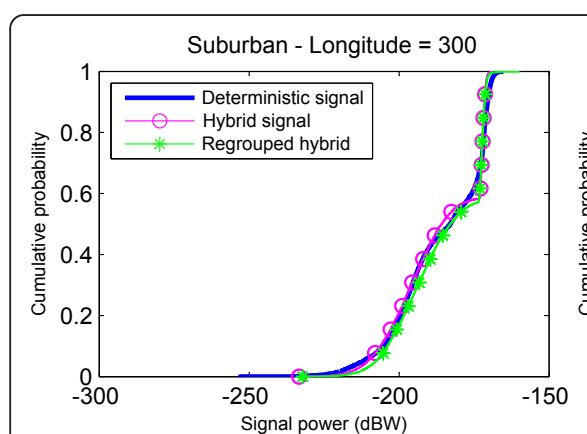

(a)

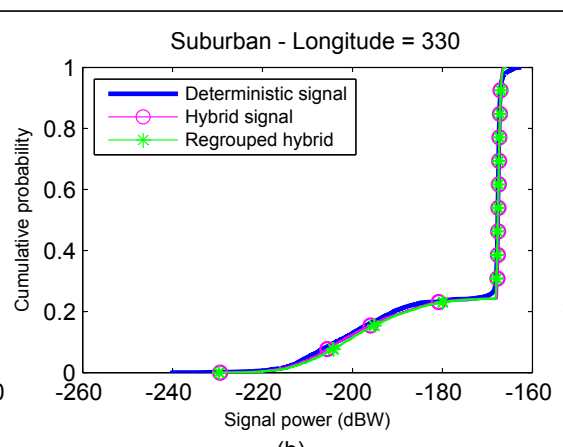

(b)

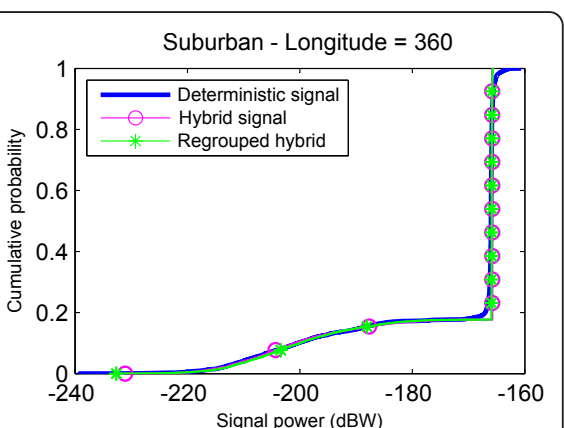

(c)

Figure 15 CDF comparison of suburban ("Empalot") simulation results: (a) $300^{\circ}$; (b) $330^{\circ}$; (c) $360^{\circ}$.

Table 4 RMS simulation errors (in dbw)

\begin{tabular}{llllll}
\hline & Dense & \multicolumn{3}{c}{ Srban } & \multicolumn{2}{c}{ Suburban } \\
\cline { 2 - 6 } & Hybrid & Hybrid & Regrouped & Hybrid & Regrouped \\
\hline $300^{\circ}$ & 3.55 & 1.83 & 1.81 & 1.54 & 1.69 \\
$330^{\circ}$ & 1.87 & 0.92 & 1.40 & 1.01 & 1.13 \\
$360^{\circ}$ & 1.58 & 0.90 & 1.47 & 0.72 & 0.80 \\
\hline
\end{tabular}


hand, seem to be slightly different. For $330^{\circ}$ and $360^{\circ}$, the RMSEs are 1.40 and 1.47 , respectively, which are greater than modelled hybrid. But for $300^{\circ}$ it is 1.81 which is slightly lower. This means the grouping of two environments may have positive or negative impact on the simulation results.

For suburban areas, the modelled hybrid curves still follow well their deterministic counterparts, we find $1.54,1.01$ and 0.72 for $300^{\circ}, 330^{\circ}$ and $360^{\circ}$, respectively. The regrouped hybrid RMSEs are higher but very close.

To conclude, the modelled hybrid gives very satisfying simulation results in terms of accuracy. The regrouped hybrid may increase or decrease the simulation error. Therefore, the grouping of urban and suburban areas is feasible and gives acceptable approximations if we seek to further simplify our model.

\subsection{Computation time evaluation}

The simulation complexity of the hybrid model can be compared to a reference model. As discussed in Section 2.2, the reference is the ray tracing software, Ergospace, in its classical configuration. Basically, in ray tracing methods, allowing more interactions (especially diffractions) greatly increases the computation time. Ergospace, for example, requires 280 seconds to simulate a course of 7,000 samples on a test machine with a Pentium IV 3.0 $\mathrm{GHz}$ processor and 2 GB RAM.

The hybrid model consists of two parts: the statistical model is very fast thanks to random generators which take only $2 \mathrm{~ms}$ to simulate 7,000 samples on the same machine. On the other hand, the deterministic model is optimized as well with a maximum of one reflection and only locates different states. This simplification efficiently reduces the computation complexity. Neither the calculation of pathloss due to interactions nor the sum of all vector multipaths at the receiver is needed. As a result, the optimized deterministic state-locating for 7,000 samples only takes $25 \mathrm{~ms}$. Combining two modules together, a gain of about $\frac{280}{(25+2) \times 10^{-3}} \approx 10370$ times can be considered in comparison to a purely deterministic model.

\section{Conclusion}

We have presented in this paper a novel hybrid LMS channel model. Working on a two- phase basis, the model combines the accuracy of a deterministic approach and the speed of a statistical one. In the learning phase, the channel behavior is modelled using adequate statistical laws with adapted parameters. These parameters were modelled by polynomial fitting and reused in similar environments in the application phase. The model is highly accurate and gives insignificant simulation errors. It is also very optimized to greatly reduce the computation time.

Our studies can be further compared to measured data for its validity, this is part of the future work of this article.

\section{Competing interests}

The authors declare that they have no competing interests.

Received: 31 August 2011 Accepted: 11 April 2012

Published: 11 April 2012

\section{References}

1. JB Keller, Geometrical theory of diffraction. J Opt Soc Am. 2(52), 116-130 (1962)

2. RG Kouyoumjian, PH Pathak, A uniform geometrical theory of diffraction for an edge in a perfectly conducting surface, in Proceedings of the IEEE, pp. 1448-1461 (1974)

3. C Loo, A statistical model for a land mobile satellite link. IEEE Trans Veh Technol. 34(3), 122-127 (1985)

4. E Lutz, D Cygan, M Dippold, F Dolainsky, W Papke, The land mobile satellite communication channel-recording, statistics, and channel model. IEEE Trans Veh Technol. 40(2), 375-386 (1991). doi:10.1109/25.289418

5. GE Corazza, F Vatalaro, A statistical model for land mobile satellite channels and its application to nongeostationary orbit system. IEEE Trans Veh Technol. 43(3), 738-742 (1994). doi:10.1109/25.312773

6. C Oestges, SR Saundersm, D Vanhoenacker-Janvier, Physical statistical modelling of the land mobile satellite channel based on ray-tracing. IEE Proc-Microw Antenn Propagat. 146, 45-49 (1999). doi:10.1049/ipmap:19990144

7. F Perez-Fontan, S Martinez, B Sanmartin, C Enjamio, P Mariño, F Machado, An enhanced Markov chain based model for the narrowband LMS channel in built-up areas. Int J Satell Commun. 23, 111-128 (2005). doi:10.1002/sat.809

8. A Abele, F Perez-Fontan, M Bousquet, P Valtr, J Lemorton, F Lacoste, E Corbel, A new physical-statistical model of the land mobile satellite propagation channel. in Proc Fourth European Conf Antennas and Propagation (EUCAP), Barcelona, Spain 1-5 (2010)

9. PR King, Modelling and Measurement of the Land Mobile Satellite MIMO Radio Propagation Channel. PhD thesis, University of Surrey (2007)

10. F Fontan, M Vazquez-Castro, C Cabado, J Garcia, E Kubista, Statistical modeling of the LMS channel. IEEE Trans Veh Technol. 50(6), 1549-1567 (2001). doi:10.1109/25.966585

11. M Berbineau, E Masson, M Chennaoui, J Marais, Satellite channel modelling using a Ray-tracing Tool for train communication, in Proc Conf 6th Int ITS Telecommunications, Chengdu, China, pp. 452-456 (2006)

12. C Tzaras, SR Saunders, BG Evans, A physical-statistical time-series model for the mobile-satellite channel, in Proceedings of 1998 IEEE APS Conference on Antennas and Propagation for Wireless Communications, IEEE, Atlanta, USA, pp. 1-4 (1998)

13. $P$ Combeau, $R$ Vauzelle, $Y$ Pousset, $L$ Aveneau, An optimization in computation time for the prediction of radio coverage zones. Radio Sci. 42 , 18 (2007)

14. JD Parsons, The Mobile Radio Propagation Channel, 2nd edn. (Wiley, West Sussex, 2000)

15. C Pereira, G Coq, X Li, Y Pousset, C Olivier, O Alata, R Vauzelle, P Combeau, Application of information criteria for the selection of the statistical fast fading model of the radio mobile channel. Int J Electron Commun (AEÜE). 64(6), 521-530 (2009)

16. M Nakagami, in The m-distribution. A general formula of intensity distribution of rapid fading, ed. by Hoffman W (Statistical Methods in Radio Wave Propagation Pergamon, Oxford, 1960), pp. 3-36

17. MF Ibrahim, JD Parsons, Signal strength prediction in built-up areas. Part 1 Median signal strength. IEEE Proc Commun Radar Signal Process. 130(5), 377-384 (1983). doi:10.1049/ip-f-1.1983.0063

18. S Saunders, B Evans, A physical-statistical model for land mobile satellite propagation in built-up areas. Proc 10th International Conference on Antennas and Propagation, Edinburgh. 2, 44-47 (1997) 
19. FP Fontan, MAV Castro, S Buonomo, JPP Baptista, BA Rastburg, S-band LMS propagation channel behavior for different environments, degrees of shadowing and elevation angles. IEEE Trans Broadcast. 44, 40-76 (1998). doi:10.1109/11.713055

doi:10.1186/1687-1499-2012-139

Cite this article as: Li et al:: Hybrid propagation channel modelling for city area land mobile satellite communications. EURASIP Journal on Wireless Communications and Networking 2012 2012:139.

\section{Submit your manuscript to a SpringerOpen ${ }^{\circ}$ journal and benefit from:}

- Convenient online submission

- Rigorous peer review

- Immediate publication on acceptance

- Open access: articles freely available online

- High visibility within the field

- Retaining the copyright to your article

Submit your next manuscript at $\gg$ springeropen.com 